\title{
Contamination, Error, and Nonspecific Molecular Tools
}

\author{
Alan T. Dyer and Kurt J. Leonard
}

First author: Department of Plant Pathology, University of Minnesota, St. Paul 55108; and second author: USDA-ARS, Cereal Disease Laboratory, St. Paul, MN 55108.

Accepted for publication 17 February 2000.

\begin{abstract}
Dyer, A. T., and Leonard, K. J. 2000. Contamination, error, and nonspecific molecular tools. Phytopathology 90:565-567.

Random amplified polymorphic DNA (RAPD) and amplified fragment length polymorphism (AFLP) are widely used in studies of genetic variation. Although it is recognized that contamination should be avoided in DNA samples, little is known about the potential hazards of low level bacterial contamination of samples from which DNA is extracted for RAPD or AFLP analyses. We found that contamination of Aphanomyces cochlioides cultures with a prokaryote at visibly undetectable levels markedly altered the results of RAPD and AFLP analyses. The contamination resulted in seven contaminant-specific RAPD products and in the sup-

pression of eight products characteristic of uncontaminated A. cochlioides cultures. Prokaryote contamination resulted in 39 contaminant-specific AFLP products, but did not cause suppression of AFLP products. Comparing $A$. cochlioides samples with outgroup A. euteiches did not clearly indicate the presence of contaminant DNA, because uneven product suppression in RAPD analysis increased the apparent similarity between contaminated samples and A. euteiches and because a high proportion of the contaminant-specific amplified products comigrated with products from A. euteiches in both RAPD and AFLP analyses. Work with organisms that are prone to contamination should employ techniques such as restriction fragment length polymorphism or DNA sequence comparisons rather than relying solely on RAPD or AFLP analyses.
\end{abstract}

Since first described in 1990 (9), random amplified polymorphic DNAs (RAPDs) have been employed in more than 1,200 reports comprising a wide range of genetic characters in bacteria, fungi, plants, and animals (Agricola Index; WinSPIRS version 4.0; SilverPlatter International, Norwood, MA). These modern markers have had a great impact in expanding the research in the genetics of organisms. They have led to molecular maps of genomes as well as to a better understanding of population dynamics and phylogenetic relationships.

Unfortunately, as valuable as RAPDs have been, they need to be applied with caution. For example, in controlled RAPD studies, it has been shown that amplified products have a suppressive effect on other potential products, such that they may display dominance in the RAPD reaction mix, a type of epistasis (3). This characteristic of RAPDs can lead to artifacts that may be difficult to detect if contaminating DNAs are not carefully excluded. Obviously, the presence of contaminant DNA can lead to additional amplification products specific to a contaminated sample, but product suppression in contaminated samples also may lead to some amplification products being specific to the uncontaminated samples. Through manipulation of the proportions of sample DNAs in a bulked composite, it was demonstrated that the proportion of a contaminant DNA needed to affect RAPD results is large (8). This suggests that, with moderate care, contaminants should not be a problem. Prokaryotes, however, have a greater DNA-to-volume ratio than do eukaryotes and they also have smaller genomes. Both of these characters increase the likelihood that an undetected prokaryotic contaminant will affect RAPD results. The important ques-

Corresponding author: A. T. Dyer; E-mail address: aland@ puccini.crl.umn.edu

The use of trade or firm names in this article is for reader information and does not imply endorsement by the United States Department of Agriculture or the University of Minnesota of any product or service.

Publication no. P-2000-0413-010

This article is in the public domain and not copyrightable. It may be freely reprinted with customary crediting of the source. The American Phytopathological Society, 2000. tion remains: "Can a bacterial contamination, slight enough to go undiscovered, still affect RAPD results?" The answer is yes. This letter describes such a situation with the sugar beet (Beta vulgaris L.) root rot pathogen Aphanomyces cochlioides Drechsler and a putative bacterial contaminant.

The study was designed to examine the genetic diversity of $A$. cochlioides in sugar beet fields. Because bacteria are often associated with cultures of Aphanomyces spp. $(1,4)$, the antibiotic rifampicin (38 mg/liter) (Sigma Chemical Co., St. Louis) was added to the V8 broth in which $A$. cochlioides isolates were grown. These cultures were allowed to grow for at least 5 days, but never exceeding 10 days to avoid possible metabolism or breakdown of the antibiotic. The mycelia were harvested in a Büchner funnel (Fisher Scientific, Pittsburgh) fitted with filter paper and a vacuum pump, and all mycelia were washed with sterile distilled water. None of the cultures displayed the milky appearance one would expect with bacterial contamination. Furthermore, many cultures were examined under the microscope at $\times 100$ magnification without detecting contamination. Once the mycelia were harvested, DNA was extracted following a standard phenol chloroform protocol (7).

Based on confirmed reproducible amplified products, the original RAPD analyses of 121 Aphanomyces isolates with six decamer primers revealed three phenotypes reported as A, B, and C (2). Phenotypes $\mathrm{A}$ and $\mathrm{C}$ were nearly identical, with a single amplified product specific to phenotype $\mathrm{C}$ distinguishing the two phenotypes (Fig. 1A) (Dice similarity $=0.987)$. (Dice similarity $=2 A /[2 A+$ $B+C$ ], in which $A$ is the number of genetic markers common to the two samples, $B$ is the number specific to sample 1 , and $C$ is the number specific to sample 2.) Phenotype B was less similar, having seven RAPD products specific to phenotype B and lacking eight RAPD products specific to phenotypes A and C (Fig. 1B). The Dice similarity coefficient for types A and B was only 0.80 . The comparison of phenotypes A and B to an outgroup species that is not pathogenic on sugar beet, A. euteiches, gave Dice similarity coefficients of 0.62 and 0.60 , respectively.

Because earlier work (5) showed that another Aphanomyces species, temporarily designated $\mathrm{A} 3$, in addition to $A$. cochlioides can infect sugar beets, it appeared that a second Aphanomyces species 
was involved in the sugar beet root rot complex. Therefore, the work was expanded to include a second form of genetic analysis, amplified fragment length polymorphism (AFLP). For this, 16 isolates were chosen, with eight isolates from RAPD phenotype A, five isolates from RAPD phenotype $\mathrm{B}$, and three isolates from RAPD phenotype C. DNAs from these isolates were examined with two selective primer pairs (MseI+TC/EcoRI+AT) and (MseI+TA/ EcoRI+AT) (Gibco-BRL, Grand Island, NY). The AFLP analysis resulted in a Dice similarity coefficient between RAPD phenotypes A and B of 0.89 , between phenotypes A and A. euteiches of 0.49 , and between RAPD phenotype B and A. euteiches of 0.47 (Fig. 2). Phenotypes A and $C$ look almost identical and have a Dice similarity coefficient of 0.98 . These results seemed normal, except that 39 products were specific to phenotype B and no products were specific to phenotypes A and C. This suggested that the DNA from phenotype B isolates might be contaminated.

To more rigorously suppress possible contaminants, isolates were regrown in V8 broth amended with an increased concentration of the antibiotic rifampicin $(50 \mathrm{mg} / \mathrm{liter})$ and the addition of the antibiotic penicillin $\mathrm{G}$ (50 mg/liter) (Sigma Chemical Co.). The new cultures were limited to 3 days of growth. Under these cultural conditions, the RAPD profiles of cultures classified as phenotype $B$ were not the same as previously observed. Instead, all of the isolates previously identified as phenotype B exhibited the phenotype A-specific product profiles. In the case of AFLP comparison, the phenotype B-specific products had disappeared. These results supported suspicions that RAPD products specific to phenotype B were artifacts of a bacterial contaminant that was eliminated either by the addition of penicillin or the increased concentration of rifampicin in the second experiment. Thus, it appears that normal precautions (7) for Aphanomyces spp. were not sufficient to prevent
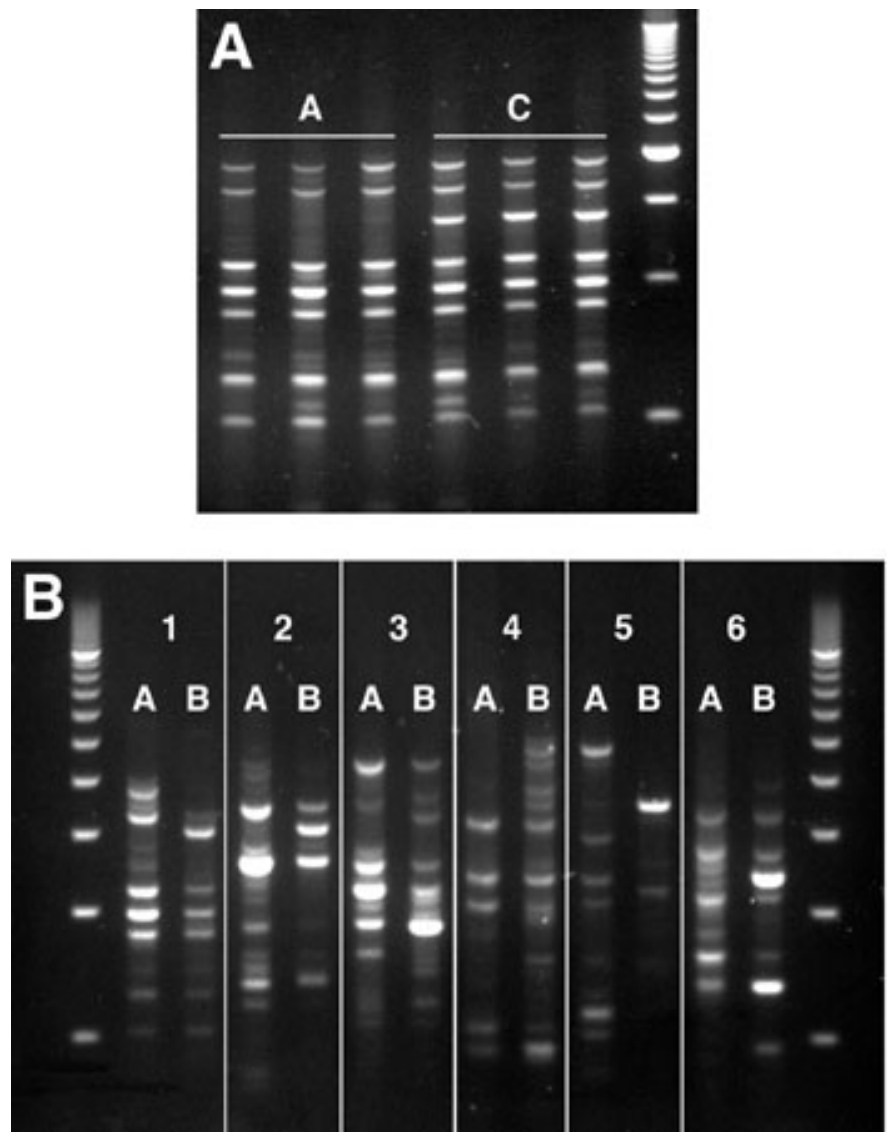

Fig. 1. A, Random amplified polymorphic DNA (RAPD) products of primer 1 for three isolates each of phenotypes $\mathrm{A}$ and $\mathrm{C}$ for Aphanomyces cochlioides. A 500-base pair (bp) ladder is on the right margin. B, RAPD products from six primers for phenotypes $\mathrm{A}$ and $\mathrm{B}$ of A. cochlioides. A 500-bp ladder is on both the left and right margins. contamination and spurious conclusions in the first RAPD comparisons with isolates of $A$. cochlioides.

In comparisons between contaminated and uncontaminated samples of the same $A$. cochlioides isolates previously examined in our RAPD analysis, no AFLP products appeared specific to the uncontaminated samples. AFLP revealed the contamination only because there was very little variability among the uncontaminated isolates. The additional products from the contaminated isolates created a recognizable, repeating pattern on a background of relatively consistent $A$. cochlioides AFLP products. If there had been greater variation in the AFLP products among the uncontaminated DNA samples, it would not have been so apparent that a specific set of products was due to a contaminant.

The following observations on the effects of the putative bacterial contaminant in our AFLP and RAPD analyses of the Aphanomyces spp. are pertinent. In the RAPD procedure, a primer may preferentially amplify one fragment of template DNA over another fragment, despite the presence of matching sites on both fragments. In effect, one potential product suppresses the amplification of another. This product-suppressive error of RAPD results in products specific to uncontaminated as well as contaminated samples, such that artifacts from contaminants resemble amplification patterns expected of normal genetic variation within Aphanomyces spp. However, in our experience, AFLP products displayed no suppressive interactions in the reaction mix, so AFLP artifacts from contaminants could be detected as products specific to the contaminant samples.

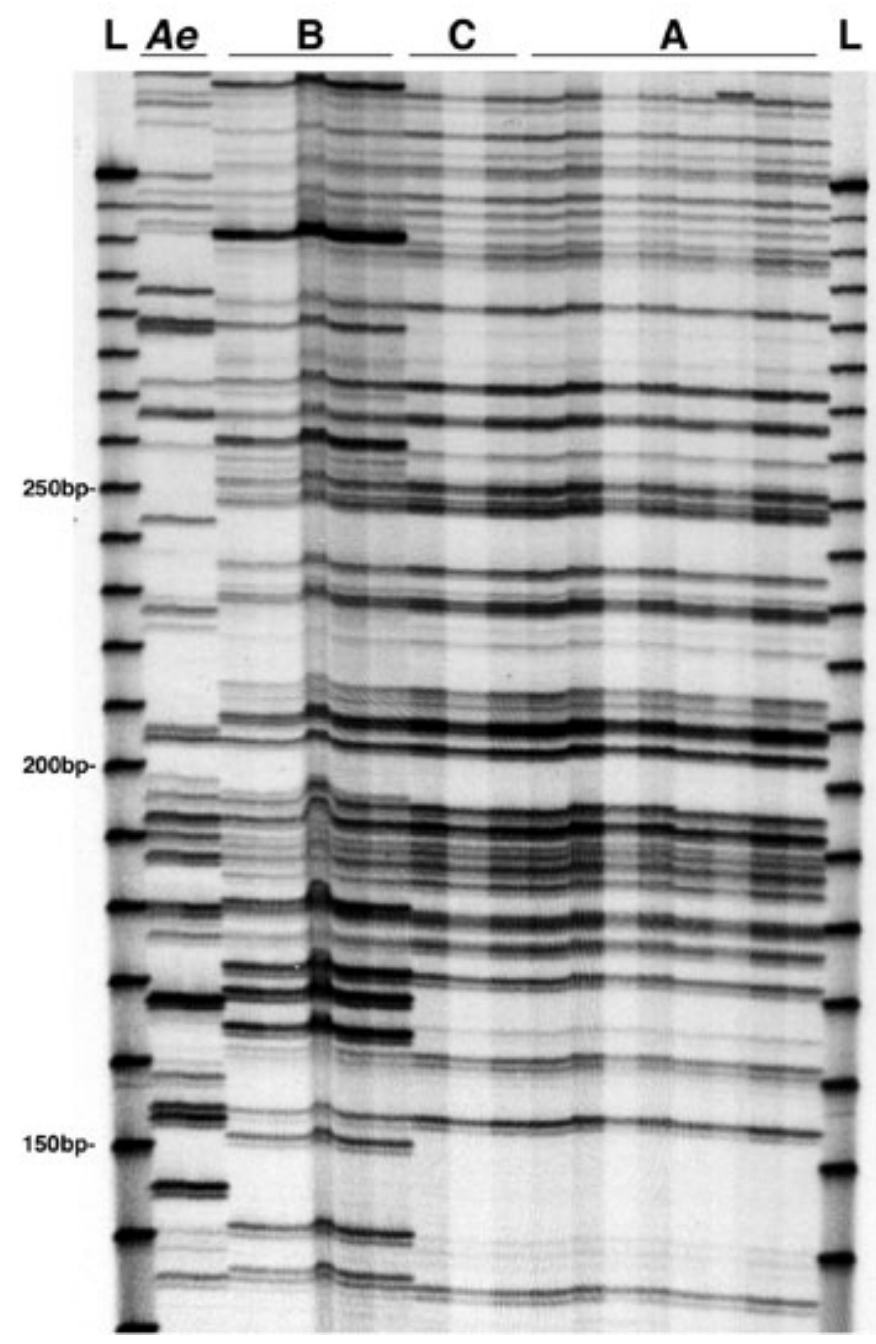

Fig. 2. Amplified fragment length polymorphic (AFLP) products of selective primer pair $M s e \mathrm{I}+\mathrm{TA} / E c o \mathrm{RI}+\mathrm{AT}$ separated on a polyacrylamide gel for Aphanomyces cochlioides isolates of phenotypes $\mathrm{B}, \mathrm{C}$, and $\mathrm{A}$. Two isolates of $A$. euteiches are designated by $A e$, and 10-base pair (bp) ladders (L) are on the left and right margins. 
It is often suggested that outgroup controls should provide evidence of contamination and that contaminated samples should be recognized by their greater genetic distances from the outgroup as reflective of the artifact banding differences. Unfortunately, disproportionate product suppression in our RAPD analyses and comigrations of nonhomologous products in both our RAPD and AFLP analyses tended to counterbalance any differences one might expect.

Theoretically, product-suppressive error might be expected to be nonselective in its effects. For example, of the 37 RAPD products from $A$. cochlioides, 21 were common to the products from $A$. euteiches and 16 were specific to $A$. cochlioides. One might expect product suppression due to contaminant DNA to effect both types of products in direct proportion to their frequency in the samples. In practice, however, the contaminant DNA suppressed a greater proportion of the A. cochlioides-specific products (5 of 16) than of common products ( 3 of 21). This disproportionate suppression of A. cochlioides-specific RAPD products tended to decrease the impact of unique products from the contaminant DNA and increase Dice similarity values between the contaminated A. cochlioides compared with A. euteiches.

Another significant aspect of the analyses with contaminated DNA was the unexpectedly high proportion of products of contaminated DNA that comigrated with products from A. euteiches in both RAPD and AFLP analyses. We concluded that the Dice similarity index values were inflated between A. euteiches and contaminated $A$. cochlioides by comigration of nonhomologous products. The basis of the conclusion is that any DNA fragment from the prokaryotic contaminant that might share conserved homology with DNA of A. euteiches should also share homology with DNA of the related species A. cochlioides. Therefore, any RAPD or AFLP products from the contaminant DNA that migrated together with the products of $A$. euteiches, but not with products from $A$. cochlioides, must represent only chance similarity of product size rather than true genetic similarity.

We found that two of seven RAPD products from the contaminated $A$. cochlioides, but not the uncontaminated $A$. cochlioides samples, comigrated with RAPD products from A. euteiches. Similarly, 8 of the 39 AFLP products from contaminated $A$. cochlioides, but not the uncontaminated A. cochlioides, comigrated with AFLP products from A. euteiches. Thus, a significant proportion of the apparent homology between the contaminated samples and $A$. euteiches samples expressed in the Dice similarity index was due to comigration of genetically unrelated products. While we cannot say with certainty how much comigration occurred between RAPD and AFLP products of uncontaminated A. cochlioides samples and A. euteiches samples, we estimate that it could account for as much as $10 \%$ of the calculated Dice similarity values for comparisons of A. cochlioides and A. euteiches.

In summary, nonspecific methods of analysis such as AFLP and RAPD must be interpreted with caution. Both methods are highly susceptible to contamination that is nearly impossible to distinguish from real variability. Due to product-suppressive errors,
RAPD artifacts resulting from contaminants are not discernible from real variation and, although AFLP was able to detect contamination in A. cochlioides, detection of contaminants with AFLP in a more genetically variable species may be masked by variation in the subject organism. We suggest that research on organisms that are often associated with bacteria should not rely on a single, nonspecific PCR-based method, or it should employ techniques such as ribosomal internal transcribed spacer sequencing, ribosomal coding gene sequencing, and restriction fragment length polymorphism to examine genetic relationships. In our experience, the outgroup A. euteiches had little or no value in the detection of contaminants of $A$. cochlioides. As additional reports on the limitations of RAPDs appear $(3,6)$, the results of nonspecific molecular methods should be reexamined. Our experience with a contaminant in seemingly clean cultures of $A$. cochlioides has led us to be much more cautious in interpreting genetic variation detected by nonspecific methods.

\section{ACKNOWLEDGMENTS}

This research was funded by the University of Minnesota, Northwest Research and Outreach Center, Crookston; the Minnesota Agricultural Experiment Station, St. Paul; the Sugarbeet Research and Education Board of Minnesota and North Dakota, Fargo; and the United States Department of Agriculture, Agricultural Research Service. We thank L. Szabo and C. Windels for all of their patience and assistance in the development of this letter.

\section{LITERATURE CITED}

1. Dreschler, C. 1929. The beet water mold and several related root parasites. J. Agric. Res. 38:309-361.

2. Dyer, A. T., Szabo, L. J., Windels, C. E., and Leonard, K. J. 1998. Population structure of Aphanomyces cochlioides in sugar beet fields (Abstr.) Phytopathology 88(suppl.):S24.

3. Hallden, C., Hansen, M., Nilsson, N. O., Hjerdin, A., and Sall, T. 1996. Competition as a source of errors in RAPD analysis. Theor. Appl. Genet. 93:1185-1192.

4. Jones, F. R., and Dreschler, C. 1925. Root rot of peas in the United States caused by Aphanomyces euteiches (n. sp.). J. Agric. Res. 30:293-325.

5. Larsson, M. 1994. Pathogenicity, morphology, and isozyme variability among isolates of Aphanomyces spp. from weeds and various crop plants. Mycol. Res. 98:231-240.

6. Lynch, M., and Milligan, B. G. 1994. Analysis of population genetic structure with RAPD markers. Mol. Ecol. 3:91-99.

7. Malvick, D. K., Grau, C. R., and Percich, J. A. 1998. Characterization of Aphanomyces euteiches strains based on pathogenicity tests and randomly amplified polymorphic DNA analyses. Mycol. Res. 102:465-475.

8. Michelemore, R. W., Paran, I., and Kesseli, R. V. 1991. Identification of markers linked to disease resistance genes by bulk segregant analysis: A rapid method to detect markers in specific genomic regions by using segregating populations. Proc. Natl. Acad. Sci. U.S.A. 88:9828-9832.

9. Williams, J. G. K., Kubelik, A. R., Livak, K. J., Rafalski, J. A., and Tingey, S. V. 1990. DNA polymorphisms amplified by arbitrary primers are useful as genetic markers. Nucleic Acids Res. 18:6531-6535. 